\title{
Female infertility due to zona pellucida defect
}

INSERM

\section{Source}

INSERM. (1999). Orphanet: an online rare disease and orphan drug data base. Female infertility due to zona pellucida defect. ORPHA:404466

Female infertility due to zona pellucida defect is a rare, genetic, female infertility disorder characterized by the presence of abnormal oocytes that lack a zona pellucida. Affected individuals are unable to conceive despite having normal menstrual cycles and sex hormone levels, as well as no obstructions in the fallopian tubes or defects of the uterus or adnexa. 